\title{
Editorial
}

\section{Advances in Catalyst Deactivation and Regeneration}

\author{
Calvin H. Bartholomew * and Morris D. Argyle \\ Chemical Engineering Department, Brigham Young University, Provo, UT 84602, USA; \\ E-Mail: mdargyle@byu.edu \\ * Author to whom correspondence should be addressed; E-Mail: calb@byu.edu; \\ Tel.: +1-801-422-4162.
}

Academic Editor: Keith Hohn

Received: 2 June 2015 / Accepted: 8 June 2015 / Published: 11 June 2015

Catalyst deactivation, the loss over time of catalytic activity and/or selectivity, is a problem of great and continuing concern in the practice of industrial catalytic processes. Costs to industry for catalyst replacement and process shutdown total tens of billions of dollars per year. While catalyst deactivation is inevitable for most processes, some of its immediate, drastic consequences may be avoided, postponed, or even reversed through regeneration.

Accordingly, there is considerable motivation to better understand catalyst decay and regeneration. Indeed, the science and technology of catalyst deactivation and regeneration have been developing rapidly as evidenced by the considerable literature addressing these topics, including about 24,000 journal articles, presentations, reports, reviews, and books; and more than 33,500 patents for the period of 1980 to 2015 . About $15 \%$ of this literature appeared in the last three years, a rate of growth double that of the past 35 years. New insights into the science of catalyst deactivation and regeneration are laying the foundation for new developments in the technology, e.g., for substantial improvements in catalyst stability and catalyst deactivation models leading to better process economics, and more effective regeneration processes.

Research and development activities in catalyst deactivation and regeneration range over a broad spectrum, which includes (1) fundamental and applied studies of deactivation and regeneration at the nano, micro, and reactor scales to understand mechanistic, process, and catalyst chemistries; (2) laboratory reactor studies of deactivation and regeneration rates to develop reaction kinetics and process variable-rate relationships important in scale-up; and (3) development of models of deactivation and regeneration processes at the catalyst surface, pellet, reactor, and process scales for controlling, optimizing, and scaling-up these processes. 
The scope of catalytic reactions in which catalyst deactivation must be addressed is wide-ranging [1-3], including homogeneous, enzymatic, heterogeneous, gas and/or liquid phase catalytic reactions in large, economically-vital industries, e.g., natural gas and petroleum refining; chemicals, foods, and pharmaceuticals production; synthetic fuels production; and air-pollution control systems in electric utility and transportation sectors. Catalysts used in these processes may undergo deactivation by one or more of the following paths or mechanisms [1,2]: (1) poisoning; (2) fouling (encapsulation of metal crystallites and plugging of pores with carbon or coke); (3) sintering; (4) reaction of active catalytic phases to inactive phases (e.g., oxidation, over-reduction, formation of metal-support compounds); (5) volatilization of active catalytic phases; and (6) mechanical failure (e.g., abrasion and/or fracture of catalyst pellets leading to plugging of pores and catalyst beds). These mechanisms are fundamentally of three types: (1) chemical; (2) thermal; and (3) mechanical. Deactivation processes [1-3] can be slow (occurring over months or years; e.g. deactivation of ammonia synthesis catalysts typically occurring over about 10 years) or very rapid (occurring over seconds or minutes, e.g., deactivation of fluid cracking catalysts, having an average lifetime of about $1 \mathrm{~s}$ ).

Regeneration processes [2,3] may involve (1) washing in various solvents, acids, or bases to remove foulants or poisons; (2) oxidative treatment in air at $300-500{ }^{\circ} \mathrm{C}$ to remove carbons or coke followed by rereduction; and (3) treatment in an oxidative atmosphere with or without halogens to redisperse sintered supported metals. These processes may be in situ or ex situ, onsite or offsite, and continuous or batch. In the case of rapid deactivation, onsite, continuous regeneration is necessary. For selected processes in which catalyst decay occurs slowly (e.g., hydrotreating or selective catalytic reduction (SCR)), spent catalysts may be shipped offsite to a vendor who specializes in regeneration and even reconstitution of catalysts (e.g., reimpregnation of the support with compounds of the active phases).

Mechanisms of deactivation and methods of preventing deactivation were addressed by Bartholomew in a comprehensive review including literature up through 2000 [1]. Fundamentals of catalyst deactivation and regeneration are reviewed in Chapter 5 of Fundamentals of Industrial Catalytic Processes, by Bartholomew and Farrauto [2] while detailed descriptions of deactivation problems in important commercial catalytic processes are summarized for each process in Chapters 6-13. Hundreds of the most pertinent, previous papers and patents addressing deactivation and regeneration up through 2005 are provided at the end of each chapter.

This special issue focuses on recent advances in catalyst deactivation and regeneration, including (1) advances in scientific understanding of deactivation and regeneration mechanisms for a wide variety of reactions, including heterogeneous gas- and liquid-phase catalytic and photocatalytic reactions; (2) development of catalysts having greater stability and reaction conditions that facilitate greater catalyst stability; and (3) examples of commercial applications based on new fundamental insights. It consists of a comprehensive review of catalyst deactivation and regeneration [3]; two topical reviews of deactivation and regeneration in Fischer-Tropsch synthesis (FTS) [4,5]; a review of Pd catalyst deactivation in emissions control converters for natural gas vehicles [6]; and four focused, topical studies of deactivation in hydrogen production in ethanol steam-reforming [7], pre-reforming of hexane [8], liquid-phase alcohol oxidation [9], and photocatalytic oxidative dehydrogenation of cyclohexane [10]. 


\section{Contribution highlights:}

1. The comprehensive review of Argyle and Bartholomew (124 pages) [3] addresses in substantive detail mechanisms of deactivation and regeneration and methods for avoiding deactivation; the previous work is documented with 384 references up through 2015. The depth of state-of-the-art commercial practice is emphasized by a detailed case study of deactivation and regeneration of SCR catalysts for reduction of $\mathrm{NO}_{x}$ from utility power boilers. A second case study briefly addresses regeneration technologies for FTS. This review should be informative reading for scientists, engineers, and managers concerned with the depth and breadth of catalyst deactivation and regeneration.

2. A "mini-review" by Rytter and Holmen [4] focuses on deactivation paths and methods of regeneration for commercial cobalt FT catalysts based mainly on patent literature. It builds on a previous, more comprehensive review of fundamental studies of deactivation and regeneration for Co FTS [11]. The authors conclude that poisoning by carbon deposits is the principal cause of long-term deactivation, in agreement with other previous studies [12-14]; the role of other deactivation mechanisms is said to be obscured by lack of data. It is emphasized that (1) deactivation rate is sensitive to reaction conditions; hence great care should be exercised in controlling process conditions (i.e., temperature, concentrations, flowrates) during start-up, steady-state operation, shutdown, and upsets; and (2) that rejuvenation and regeneration of Co catalysts are economic necessities for extending catalyst life to several years.

3. A review by Jacobs et al. [5] addresses the influence of crystallite diameter, Co loading, Co spatial uniformity, and reduction promoters on stability of Co FTS. Consistent with the primary focus of this review on the role of noble metal promoters, substantial activity and selectivity data are reported for cobalt catalysts promoted with $\mathrm{Ag}, \mathrm{Au}, \mathrm{Cu}, \mathrm{Pt}, \mathrm{Pd}, \mathrm{Re}$, and $\mathrm{Ru}$ over a range of promoter concentrations and are supplemented with selective XAFS/XANES studies. While Pt, Re, and Ru promoters at commercially viable levels $(0.05-0.5$ wt.\%) do not influence intrinsic Co catalyst activity, $\mathrm{Cu}$ (and $\mathrm{Ag}$ to a much lesser extent) apparently poison Co in FTS. Noble metals of high hydrogenation activity, i.e., $\mathrm{Pd}$ and $\mathrm{Cu}$ promote unfavorably high methane selectivities. Computational studies predict that Pt or Ru impede Co activity decay caused by carbon deposits on the Co surface by increasing the energy barrier for carbon-carbon coupling reactions. Indeed, experimental studies show that less carbon is formed on Co catalysts containing these promoters [1]. Pt-, Re-, and Ru-promoted Co can be regenerated at least 3 times; Pt-promoted Co improves its reducibility after 3 cycles. By contrast, Au is separated from Co after only one cycle.

4. Gholami et al. [6] review studies of deactivation of Pd catalysts by water during low-temperature oxidation of $\mathrm{CH}_{4}$ in converters for natural gas vehicles. $\mathrm{CH}_{4}$ oxidation in a $\mathrm{Pd}$ catalytic converter for a natural gas engine is limited by low exhaust gas temperatures $\left(500-550{ }^{\circ} \mathrm{C}\right.$ ) and low concentrations of $\mathrm{CH}_{4}(400-1500$ ppmv) that must be reacted in the presence of large quantities of $\mathrm{H}_{2} \mathrm{O}(10-15 \%)$ and $\mathrm{CO}_{2}(15 \%)$, under transient exhaust gas flows, temperatures, and compositions. While activities of $\mathrm{Pd}$ catalysts are highest relative to other catalysts for $\mathrm{CH}_{4}$ oxidation, under converter conditions $\mathrm{Pd}$ loses activity due to reaction inhibition by $\mathrm{H}_{2} \mathrm{O}$ below $500{ }^{\circ} \mathrm{C}$ and $\mathrm{H}_{2} \mathrm{O}$ induced sintering above $500{ }^{\circ} \mathrm{C}$. Water inhibition is postulated to occur by either formation of relatively stable $\mathrm{Pd}(\mathrm{OH})_{2}$ and/or partial blocking by $\mathrm{OH}$ groups of the $\mathrm{O}$ exchange between the 
support and Pd active sites. Evidence from FTIR and isotopic labeling favors the latter route. Addition of $\mathrm{Rh}$ or $\mathrm{Pt}$ to $\mathrm{Pd}$ and use of supports of high $\mathrm{O}$ mobility (e.g., $\mathrm{CeO}_{2}$ ) improve catalyst activity and stability.

5. Effects of $\mathrm{Ce}$ and $\mathrm{Zr}$ addition at different $\mathrm{Zr} / \mathrm{Ce}$ mass ratios on the performance and stability of $\mathrm{Ni} / \mathrm{SiO}_{2}$ catalysts for $\mathrm{H}_{2}$ production via ethanol steam reforming were studied by Calles et al. [7]. Incorporation of $\mathrm{Ce}$ or $\mathrm{Zr}$ in $\mathrm{Ni} / \mathrm{SiO}_{2}$ facilitates complete ethanol conversion with time, otherwise not possible in their absence due to coke formation. With incorporation of both $\mathrm{Ce}$ and $\mathrm{Zr}$ into $\mathrm{SiO}_{2}$ before addition of $\mathrm{Ni}$, a $\mathrm{Ce}_{x} \mathrm{Zr}_{1-x} \mathrm{O}_{2}$ solid solution is formed which interacts more strongly with $\mathrm{Ni}$ than $\mathrm{SiO}_{2}$, facilitating improved $\mathrm{Ni}$ dispersion and thermal stability. While activities and stabilities of all Ni/Ce $\mathrm{Zr}_{1-x} \mathrm{O}_{2} / \mathrm{SiO}_{2}$ catalysts after $8 \mathrm{~h}$ of reaction at $600{ }^{\circ} \mathrm{C}$ are desirably high, the highest hydrogen selectivity was realized for a $\mathrm{Zr} / \mathrm{Ce}$ mass ratio of three.

6. Trunfio and Arena [8] studied deactivation of a model Ni/MgO catalyst in the pre-reforming of n-hexane at $450^{\circ} \mathrm{C}$ and 5-15 bar at steam-to-carbon (S/C) ratios of 1.5-3.5. Fouling of the catalyst by coke causes a first-order, exponential decay in activity. While $\mathrm{H}_{2}$ substantially hinders coke formation, it also facilitates minor sintering of the active Ni phase. Poisoning of nickel by thiophene causes a rapid linear drop in activity. Rate constants obtained from comparison of log activity vs time patterns at different conditions enable (1) assessment of the relative effects of pressure, S/C ratio, $\mathrm{H}_{2}$ concentration, and sulfur content on deactivation rate and (2) determination of deactivation rate constants for the stoichiometric equations, i.e., hexane conversion, methanation, gasification, and water-gas-shift during pre-reforming.

7. Skupien et al. [9] investigated the reversible inhibition of gold-catalyzed benzyl-alcohol oxidation to benzaldehyde in a liquid-phase, batch reactor. The reaction was carried out in liquid toluene in the presence of entrained air at $80{ }^{\circ} \mathrm{C}$. Since this oxidation also requires a small amount of water to be present during reaction, effects of water were investigated. Liquid-phase species were analyzed by GC, while species adsorbed on Au were determined by Diffuse Reflectance FTIR (DRIFTS). Benzyl-benzoate is a significant byproduct. Benzoic acid, produced at a very low concentration $\left(<0.2 \mu \mathrm{mol} / \mathrm{g}_{\text {liq }}\right)$ during reaction, was identified to be present on the Au surface using DRIFTS; either the acid, or a product of the acid, is postulated to be the principal inhibiting species or poison; it apparently adsorbs strongly on the Au surface to high coverages. Addition of $\mathrm{K}_{2} \mathrm{CO}_{3}$ or $\mathrm{KF}$ reverses inhibition by neutralizing benzoic acid, while addition of a small quantity of water (e.g., $0.5 \mathrm{~g} / \mathrm{g}_{\text {cat }}$ ) enhances this effect. However, basic conditions also cause a decrease in selectivity to benzaldehyde and an increase in ester production. The inhibition and its reversal by base are described quantitatively by a robust, comprehensive kinetic model which predicts observed species concentrations with time.

8. A study of catalyst deactivation in the gas-phase, photocatalytic cyclohexane oxidative dehydrogenation on $\mathrm{MoO}_{x} / \mathrm{SO}_{4} / \mathrm{TiO}_{2}$ catalysts was carried out by Vaiano et al. [10]. Mass spectrometer and IR analysis of gas phase products at the exit of a fixed bed photoreactor was coupled with in situ monitoring of the photocatalyst surface using DRIFTS. From DRIFTS analysis, the formation of an organo-sulfur compound from the catalyst sulfate is inferred, which in the absence of irradiation initiates hydrogen abstraction of cyclohexane, thereby enhancing cyclohexane 
adsorption; following irradiation, the greater concentration of adsorbed cyclohexane increases the dehydrogenation rate. However, the accompanying stepwise reduction of the sulfate by protons causes formation of $\mathrm{SO}_{2}$ and a loss of surface sulfur, which in turn causes a decrease in photocatalytic activity during irradiation. The loss of sulfur, however, improves product selectivity, since benzene yield decreases with decreasing sulfate coverage. During irradiation, an additional, strong deactivation is observed due to the poisoning of the surface by carbon deposits strongly adsorbed on catalyst surface.

The Editors thank Keith Hohn, Editor-in-Chief for the opportunity to organize this special issue and Mary Fan, Senior Assistant Editor, and the staff of the Catalysts Editorial Office for their significant support. CHB is especially grateful to Mary Fan for her patience, understanding, and encouragement in the face of substantial delays on his part due to health challenges and a family tragedy. Furthermore, we would like to thank the reviewers of the submitted manuscripts for their recommendations and the contributing authors for their hard work in revising their manuscripts several times in order to meet the high standards of this special issue. The high quality of the published work appears to have rewarded these efforts.

\section{References}

1. Bartholomew, C.H. Mechanisms of catalyst deactivation. Appl. Catal. A 2001, 212, 17-60.

2. Bartholomew, C.H.; Farrauto, R.J. Fundamentals of Industrial Catalytic Processes, 2nd ed.; Wiley-Interscience: Hoboken, NJ, USA, 2006.

3. Argyle, M.D.; Bartholomew, C.H. Heterogeneous Catalyst Deactivation and Regeneration: A Review. Catalysts 2015, 5, 145-269.

4. Rytter, E.; Holmen, A. Deactivation and Regeneration of Commercial Type Fischer-Tropsch Co-Catalysts-A Mini-Review. Catalysts 2015, 5, 478-499.

5. Jacobs, G.; Ma, W.; Davis, B.H. Influence of Reduction Promoters on Stability of Cobalt/ $\gamma$-Alumina Fischer-Tropsch Synthesis Catalysts. Catalysts 2014, 4, 49-76.

6. Gholami, R.; Alyani, M.; Smith, K.J. Deactivation of Pd Catalysts by Water during Low Temperature Methane Oxidation Relevant to Natural Gas Vehicle Converters. Catalysts 2015, 5, 561-594.

7. Calles, J.A.; Carrero, A.; Vizcaíno, A.J.; Lindo, M. Effect of Ce and $\mathrm{Zr}$ Addition to Ni/SiO 2 Catalysts for Hydrogen Production through Ethanol Steam Reforming. Catalysts 2015, 5, 58-76.

8. Trunfio, G.; Arena, F. Deactivation Pattern of a "Model" Ni/MgO Catalyst in the Pre-Reforming of n-Hexane. Catalysts 2014, 4, 196-214.

9. Skupien, E.; Berger, R.J.; Santos, V.P.; Gascon, J.; Makkee, M.; Kreutzer, M.T.; Kooyman, P.J.; Moulijn, J.A.; Kapteijn, F. Inhibition of a Gold-Based Catalyst in Benzyl Alcohol Oxidation: Under-standing and Remediation, Catalysts 2014, 4, 89-115.

10. Vaiano, V.; Sannino, D.; Almeida, A.R.; Mul, G.; Ciambelli, P. Investigation of the Deactivation Phenomena Occurring in the Cyclohexane Photocatalytic Oxidative Dehydrogenation on $\mathrm{MoO}_{x} / \mathrm{TiO}_{2}$ through Gas Phase and in situ DRIFTS Analyses. Catalysts 2013, 3, 978-997. 
11. Tsakoumis, N.E.; Rønning, M.; Borg, O.; Rytter, E.; Holmen, A. Deactivation of cobalt based Fischer-Tropsch catalysts: A review. Catal. Today 2010, 154, 162-182.

12. Saib, A.M.; Moodley, D.J.; Ciobica, I.M.; Hauman, M.M.; Sigwebela, B.H.; Weststrate, C.J.; Niemantsverdriet, J.W.; van de Loosdrecht, J. Fundamental understanding of deactivation and regeneration of cobalt Fischer-Tropsch synthesis catalysts. Catal. Today 2010, 154, 271-282.

13. Argyle, M.D.; Frost, T.S.; Bartholomew, C.H. Modeling cobalt Fischer-Tropsch catalyst deactivation with generalized power law expressions. Top. Catal. 2014, 57, 415-429.

14. Keyvanloo, K.; Fisher, M.J.; Hecker, W.C.; Lancee, R.J.; Jacobs, G.; Bartholomew, C.H. Kinetics of deactivation by carbon of a cobalt Fischer-Tropsch catalyst: Effects of $\mathrm{CO}$ and $\mathrm{H}_{2}$ partial pressures. J. Catal. 2015, 327, 33-47.

(C) 2015 by the authors; licensee MDPI, Basel, Switzerland. This article is an open access article distributed under the terms and conditions of the Creative Commons Attribution license (http://creativecommons.org/licenses/by/4.0/). 